\title{
Numerical Treatment of Fractional Tumor Immune System Model by Adam-Bashforth Moulton Method
}

\author{
${ }^{1}$ Majid M. Hamed, ${ }^{2}$ Ali F. Jameel, ${ }^{3}$ N.R. Anakira, ${ }^{2}$ A. Saaban and ${ }^{1}$ Farah A. Abdullah \\ ${ }^{1}$ School of Mathematical Sciences, University Science Malaysia (USM), 11800 Penang, Malaysia \\ ${ }^{2}$ School of Quantitative Sciences, College of Art and Sciences, Universiti Utara Malaysia (UUM), \\ Sintok, 06010 Kedah, Malaysia \\ ${ }^{3}$ Department of Mathematics, Faculty of Science and Technology, Irbid National University, \\ 2600 Irbid, Jordan
}

\begin{abstract}
In this study, the fractional order of tumor cell growth and their interactions with effectors such as cytotoxic T-cells, $\mathrm{E}_{1}(\mathrm{t})$ and natural killer cells $\mathrm{E}_{2}(\mathrm{t})$ are studied. The Adam-Bashforth-Moulton algorithm is used to numerically solve and simulate the model of the tumor immune system with fractional order. From the observation of the numerical result, reduces $\alpha$ from 1-0.75, decrease the maximum of tumor cells. Numerical simulations illustrate that the fractional-order derivative enriches the dynamics of the system and increases the complexity of the observed behaviors which confirms that the fractional-order may play the role of memory in the system.
\end{abstract}

Key words: Fractional calculus, Adam-Bashforth-Moulton method, tumor-immune system, illustrate, memory, numerical simulations

\section{INTRODUCTION}

Tumor and cancer are two different words but have a relationship. As we talk about cancer, we also need to know the origin of cancer. Cancer is a term for diseases in which abnormal cells divide without control and can invade nearby tissues (Arshad et al., 2016). On the other hand, the tumors are groups of abnormal cells that form lumps or growths. Different types of tumors grow and behave differently, depending on whether they are non-cancerous (benign) or cancerous (malignant). It is reported that cancer is the second leading cause of death globally and accounted for 8.8 million deaths in 2015 . Globally, nearly 1 in 6 deaths is due to cancer (Anonymous, 2018). There are over 200 different cancers including breast cancer, skin cancer, lung cancer, ovarian cancer, brain cancer, colon cancer, prostate cancer and lymphoma cancer. In the last two decades, a lot of mathematical models for cancer have been proposed. Mathematical modelling in cancer research helps to elucidate mechanisms and by providing quantitative predictions that can be validated (Ahmed and El-Saka, 2010). The recent expansion of quantitative models addresses many questions regarding tumor initiation, progression and metastases as well as intra-tumor heterogeneity, treatment responses and resistance.
Mathematical models can complement experimental and clinical studies but also challenge current paradigms, redefine our understanding of the mechanisms driving tumorigenesis and shape future research in Cancer Biology (Altrock et al., 2015; Itik and Banks, 2010). The cancer research focusing on tumor growth control has reached a remarkable attention (Sohail et al., 2015). However, there are few studies focusing on the tumor in the fractional order model (Rihan, 2013; Sohail et al., 2015; Rihan et al., 2016). The motivation comes from the observation that fractional model can have better feature in explaining certain conditions in cancer modelling such as hereditary properties. We also extend the model solution into other numerical schemes to observe the progression behavior of the cells. Numerical solutions of tumor-immune system with fractional order is calculated by using a predictor-corrector method (Majid and Suleiman; Iori et al., 1999). The derivative is defined in the Caputo derivative fractional sense (Caponetto et al., 2010; Abdeljawad, 2011; Almeida, 2017). Numerical examples illustrate that the predictor-corrector method can be applied to solve the fractional order of tumor-immune system simply and effectively. Numerical simulations show that the fractional-order derivative enriches the system and increases the complexity of the observed behaviors which confirms that the fractional-order may

Corresponding Author: Ali F. Jameel, School of Quantitative Sciences, College of Art and Sciences, Universiti Utara Malaysia (UUM), Sintok, 06010 Kedah, Malaysia 
play with the memory of the system (Rihan et al., 2016). The aim of this research isto introduce a study of the tumor-immune system with fractional-order by using PECE scheme. The PECE (Predict, Evaluate, Correct, Evaluate) is a numerical method that uses predictor-corrector algorithm or known Adams-Bashforth-Moulton method to approximate the system. By using MATLAB Software, the simulation of predictor corrector method can be formed. In our research, the interval of fractional order parameter is range from $0-1$.

\section{MATERIALS AND METHODS}

Tumor immune system model: In this study, our focus is on observing numerical scheme simulation result from fractional tumor immune system. The model can be generally expressed by:

$$
\left\{\begin{array}{c}
D^{\alpha} E_{1}(t)=-d_{1} E_{1}(t)+\frac{C^{2}(t) E_{1}(t)}{C^{2}(t)+K_{1}} \\
D^{\alpha} C(t)=m C(t)-r_{1} C(t) E_{1}(t)-r_{2} C(t) E_{2}(t) \\
D^{\alpha} E_{2}(t)=-d_{2} E_{2}(t)+\frac{C^{2}(t) E_{2}(t)}{C^{2}(t)+K_{2}}
\end{array}\right.
$$

where, $0<\alpha \leq 1$ (Rihan et al., 2016). Initially, the model has been discussed by Rihan et al. (2016), they investigated the impact of effectors to the cancer cells. The model can be grouped in three parts which are $\mathrm{E}_{1}, \mathrm{E}_{2}$ effectors cells and cancer cells. The parameter $\mathrm{d}_{1}$ represents natural decay rate of the effect cells $E_{1}(t)$. The $k_{1}$ and $k_{2}$ parameters denotes half saturation and $\mathrm{m}$ is the growth rate of tumor cells. Further elaboration on model 1 will be given later.

Predictor-corrector scheme: In this study, an algorithm for the numerical solution of fractional differential equation in Caputo type will be presented. Generally, a time fractional differential equation can be written as:

$$
D_{t}^{\alpha} y(t)=f(t, y(t))
$$

with initial condition:

$$
\mathrm{y}^{(\mathrm{k})}(0)=\mathrm{y}_{0}^{(\mathrm{k})}, \mathrm{k}=0,1,2, \ldots, \mathrm{m}-1
$$

where, $\alpha$ refers to Caputo fractional differential operator for $0<\alpha \leq 1$. The algorithm is based on the fractional formulation of the classical predictor-corrector method to solve the fractional differential equation of Caputo type in specific by using the formulation of the Volterra integral equation form, i.e., (Diethelm, 2010):

$$
y(t)=\sum_{k=0}^{m-1} y_{0}^{(k)} \frac{t^{k}}{k !}+\frac{1}{\Gamma(\alpha)} \int_{0}^{t}(t-t)^{\alpha-1} f(t, y(z)) d z
$$

In order to develop the numerical method, the classical one-step of predictor-corrector algorithm for first-order must be derived before formulation of fractional numerical method. Assume that the initial value problem for the first order differential equation given by:

$$
D y(t)=f(t, y(x))
$$

$$
\mathrm{y}(0)=\mathrm{y}_{0}
$$

Function $\mathrm{f}$ assumed has a unique solution exists on some interval $[0, T]$. Following by Diethelm (2010) he used the predictor-corrector technique on uniform grid $\left\{\mathrm{t}_{\mathrm{n}}=\mathrm{nh}\right.$ : $\mathrm{n}=0,1, \ldots, \mathrm{N}\}$ with some integer $\mathrm{N}$ and $\mathrm{h}=\mathrm{T} / \mathrm{N}$. The approximation can be written as $Y_{j} \approx y\left(t_{j}\right),(j=1,2, \ldots, n)$. The approximation $\mathrm{y}_{\mathrm{n}+1}$ means:

$$
y\left(t_{n+1}\right)=y\left(t_{n}\right)+\int_{t_{n}}^{t_{n+1}} f(z, y(z)) d z
$$

This equation follows upon integration of Eq. 5 on the interval $\left[t_{n}, t_{n+1}\right]$ without knowing either of the expressions on the right-hand side of Eq. 7 exactly. However, there is an approximation for $y\left(t_{n}\right)$, namely $y_{n}$ that can be used. The integral is then replaced by the two-point trapezoidal quadrature formula given by:

$$
\int_{a}^{b} g(z) d z \approx \frac{b-a}{2}(g(a)+g(b))
$$

Thus, giving an equation for the unknown approximation $\mathrm{y}_{\mathrm{n}+1}$ :

$$
y_{n+1}=y_{n}+\frac{t_{n+1}-t_{n}}{2}\left[f\left(t_{n}, y\left(t_{n}\right)\right)+f\left(t_{n+1}, y\left(t_{n+1}\right)\right)\right]
$$

The $y\left(t_{n}\right)$ and $y\left(t_{n+1}\right)$ are replaced by their approximations $\mathrm{y}_{\mathrm{n}}$ and $\mathrm{y}_{\mathrm{n}+1}$, respectively which later produces the equation for the implicit one-step predictor-corrector method, given by:

$$
\mathrm{y}_{\mathrm{n}+1}=\mathrm{y}_{\mathrm{n}}+\frac{\mathrm{t}_{\mathrm{n}+1}-\mathrm{t}_{\mathrm{n}}}{2}\left[\mathrm{f}\left(\mathrm{t}_{\mathrm{n}}, \mathrm{y}_{\mathrm{n}}\right)+\mathrm{f}\left(\mathrm{t}_{\mathrm{n}+1}, \mathrm{y}_{\mathrm{n}+1}\right)\right]
$$

Diethelm (2010) advises that the predictor or preliminary approximation of $\mathrm{y}_{\mathrm{n}+1}^{\mathrm{p}}$ can be obtained by only replacing the trapezoidal quadrature formula in the rectangle rule giving that the explicit forward Euler method to produce the following Eq. 10: 
and:

$$
\mathrm{y}_{\mathrm{n}+1}^{\mathrm{p}}=\mathrm{y}_{\mathrm{n}}+\mathrm{hf}\left(\mathrm{t}_{\mathrm{n}}, \mathrm{y}_{\mathrm{n}}\right)
$$

$$
y_{n+1}=y_{n}+\frac{h}{2}\left[f\left(t_{n}, y_{n}\right)+f\left(t_{n+1}, y_{n+1}^{p}\right)\right]
$$

This approach is known as the one-step of predictor-corrector method.

Fractional derivative formulation: From the classical algorithm, it is possible to make modification to form fractional-order problem. The aim is to derive an equation which is similar to equation in Eq. 7 but the range of integration now starts at 0 instead of $\mathrm{t}_{\mathrm{n}}$ (Diethelm et al., 2002). The trapezoidal quadrature formula is used to replace the integral with $\mathrm{t}_{j}\{\mathrm{j}=0,1, \ldots, \mathrm{n}+1\}$ and interpret the weight function $\left(t_{n+1}\right)^{\alpha-1}$ by using the approximation given by:

$$
\int_{0}^{t_{n+1}}\left(t_{n+1}-z\right)^{\alpha-1} g(z) d z \approx \int_{0}^{t_{n+1}}\left(t_{n+1}-z\right)^{\alpha-1} g_{n+1}^{*}(z) d z
$$

where, $\mathrm{g}^{*}{ }_{\mathrm{n}+1}$ is any intermediate value read of from straight line for $\mathrm{g}$. Based on the quadrature theory, the integral on the right-hand side of Eq. 12 can be written as:

$$
\int_{0}^{t_{n+1}}\left(t_{n+1}-z\right)^{\alpha-1} g_{n+1}^{*}(z) d z=\frac{h^{\alpha}}{\alpha(\alpha+1)} \sum_{j=0}^{n+1} a_{j, n+1} g\left(t_{j}\right)
$$

Where:

$$
a_{j, n+1}=\left\{\begin{array}{cc}
n^{\alpha+1}-(n-\alpha)(n+1)^{\alpha} & j=0, \\
(n-j+2)^{\alpha+1}+(n-j)^{\alpha+1}-2(n-j+1)^{\alpha+1}, & 1 \leq j \leq n \\
1, & j=n+1
\end{array}\right.
$$

This gives us the corrector formula for fractional predictor-corrector method, given by:

$$
\begin{aligned}
& y^{c}\left(t_{n+1}\right)=\sum_{k=0}^{[\alpha]-1} y_{0}^{(j)} \frac{t_{n+1}^{k}}{k !}+\frac{h^{\alpha}}{\Gamma(\alpha+2)} f\left(t_{n+1}, y_{h}^{p}\left(t_{n+1}\right)\right)+ \\
& \frac{h^{\alpha}}{\Gamma(\alpha+2)} \sum_{j=0}^{n} a_{j, n+1} f\left(t_{j}, y\left(t_{j}\right)\right)
\end{aligned}
$$

For the predictor formula, calculation of the value $\mathrm{y}_{\mathrm{h}}^{\mathrm{p}}\left(\mathrm{t}_{\mathrm{n}+1}\right)$ is required. Similar concept was used for predictor-corrector technique to generalize the one-step predictor-corrector method by replacing the integral with the product of rectangle rule:

$$
\int_{0}^{t_{n+1}}\left(t_{n+1}-z\right)^{\alpha-1} g(z) d z \approx \sum_{j=0}^{n} b_{j, n+1} g\left(t_{j}\right)
$$

Where:

$$
b_{j, n+1}=\frac{h^{\alpha}}{a}\left((n-j+1)^{\alpha}-(n-j)^{\alpha}\right), 0 \leq j \leq n
$$

Thus, the predicted value $y_{h}^{p}\left(t_{n+1}\right)$ is determined by the fractional predictor-corrector method:

$$
\mathrm{y}_{\mathrm{h}}^{\mathrm{p}}\left(\mathrm{t}_{\mathrm{n}+1}\right)=\sum_{\mathrm{k}=0}^{\mathrm{m}-1} \mathrm{y}_{0}^{(\mathrm{k})} \frac{\mathrm{t}_{\mathrm{n}+1}^{\mathrm{k}}}{\mathrm{k} !}+\frac{1}{\Gamma(\alpha)} \sum_{\mathrm{j}=0}^{\mathrm{n}} \mathrm{b}_{\mathrm{j}, \mathrm{n}+1} \mathrm{f}\left(\mathrm{t}_{\mathrm{j}}, \mathrm{y}_{\mathrm{h}}\left(\mathrm{t}_{\mathrm{j}}\right)\right)
$$

The basic algorithm of the fractional predictor-corrector method is described in Eq. 15 and 17 with weights $a_{j, n+1}$ and $b_{j, n+1}$ defined in 14 and 16 , respectively.

Fractional model of tumor-immune system: Immune system is considered as one of the most attractive schemes in terms of Biology and Mathematics (Mophou and Fotsing, 2014; Bellomo, 2000; Bellomo et al., 2008; Ferdi, 2012). Immune system is multi-functional with several metabolic pathways, therefore, most effector cells achieve more than one function. In addition, each function of the immune system is typically done by more than one effector which makes it more complex. Based on Rihan et al. (2016) the model has includes two immune effectors: $E_{1}(t)$ and $E_{2}(t)$ (such as cytotoxic T-cells and natural killer cells) interacting with the cancer cells, $\mathrm{C}(\mathrm{t})$. The model takes the form:

$$
\begin{aligned}
& D^{\alpha} E_{1}(t)=-d_{1} E_{1}(t)+\frac{C^{2}(t) E_{1}(t)}{C^{2}(t)+K_{1}} \\
& D^{\alpha} C(t)=m C(t)-r_{1} C(t) E_{1}(t)-r_{2} C(t) E_{2}(t) \\
& D^{\alpha} E_{2}(t)=-d_{2} E_{2}(t)+\frac{C^{2}(t) E_{2}(t)}{C^{2}(t)+K_{2}}
\end{aligned}
$$

where, $0<\alpha \leq 1$. The parameter represents natural decay rate of the effect cells $E_{1}(t) . k_{2}$ and $k_{2}$ are half saturation parameters, $m$ is the growth rate of tumor cells. $r_{2}$ and $r_{2}$ are the reduction rate of tumor cells due to presence of the effector cells $\mathrm{E}_{1}(\mathrm{t})$ and $\mathrm{E}_{2}(\mathrm{t})$, respectively. The parameter $\mathrm{d}_{2}$ represents natural decay rate of the effect cells $\mathrm{E}_{2}(\mathrm{t})$. All of these parameters are supposed to be positive constants. The interaction terms in the first and third equations of model satisfy the cross-reactivity property of the immune system. It has been assumed that $\left(\mathrm{d}_{1} \mathrm{k}_{1} /\left(1-\mathrm{d}_{1}\right)\right)<<\left(\mathrm{d}_{2} \mathrm{k}_{2} /(1-\right.$ $\left.\mathrm{d}_{1}\right)$ ) to avoid the non-biological interior solution where both immune effectors coexist.

Estimation of parameters: The parameter values and initial conditions used for the analysis and numerical solutions to the system of fractional equations are shown in Table 1 (Rihan et al., 2016). 
Table 1: Parameters and values of the system of fractional differential equation

\begin{tabular}{ll}
\hline Parameters & Values \\
\hline$\alpha$ & $1,0.75,0.5,0.25$ \\
$\mathrm{~m}$ & 1 \\
$\mathrm{r}_{1}$ & 1 \\
$\mathrm{r}_{2}$ & 1 \\
$\mathrm{~d}_{1}$ & 0.3 \\
$\mathrm{~d}_{2}$ & 0.7 \\
$\mathrm{k}_{1}$ & 0.3 \\
$\mathrm{k}_{1}$ & 0.7 \\
\hline
\end{tabular}

Simulations: In this study, we present numerical simulations of Eq. 2. Since, most of the fractional order differential equations do not have exact analytical solutions, numerical techniques and approximation will be used. Diethelm et al. (2002) used a predictor-corrector method to find numerical solution of fractional differential equation. The method may be used both for linear and nonlinear problems. Since, this dissertation investigate the mathematical model of tumor-immune in nonlinear equations, therefore, predictor-corrector algorithm will applied to the fractional order tumor-immune system model by discretizing the grid as $\mathrm{h}=\mathrm{T} / \mathrm{N}, \mathrm{t}_{\mathrm{n}}=\mathrm{nh}$ and $\mathrm{n}=0,1,2, \ldots, \mathrm{N}^{+}$, system of model in Eq. 3 can be discretized as follows: the following is structure where $\mathrm{E}_{1}^{\mathrm{p}}$, $\mathrm{E}^{\mathrm{p}}{ }_{2}$ and $\mathrm{C}^{\mathrm{p}}$ referring to predictor formulation scheme:

$$
\begin{aligned}
& E_{1, n+1}^{C}=E_{1}(0)+\frac{h^{a}}{\Gamma(a+2)}\left(-d_{1} E_{1, n+1}^{p}+\frac{\left(C_{n+1}^{p}\right)^{2} E_{1, n+1}^{p}}{\left(C_{n+1}^{p}\right)^{2}+k_{1}}\right)+ \\
& \frac{h^{\alpha}}{\Gamma(\alpha+2)} \sum_{j=0}^{n} a_{j, n+1}\left(-d_{1} E_{1, j}+\frac{\left(C_{j}\right)^{2} E_{1, j}}{\left(C_{j}\right)^{2}+k_{1}}\right) \\
& E_{2, n+1}^{c}=E_{2}(0)+\frac{h^{a}}{\Gamma(a+2)}\left(-d_{2} E_{2, n+1}^{p}+\frac{\left(C_{n+1}^{p}\right)^{2} E_{2, n+1}^{p}}{\left(C_{n+1}^{p}\right)^{2}+k_{1}}\right)+ \\
& \frac{h^{a}}{\Gamma(\alpha+2)} \sum_{j=0}^{n} a_{j, n+1}\left(-d_{2} E_{2, j}+\frac{\left(C_{j}\right)^{2} E_{2, j}}{\left(C_{j}\right)^{2}+k_{2}}\right)^{c} \\
& C_{n+1}^{c}=C(0)+\frac{h^{a}}{\Gamma(\alpha+2)}\left(m_{n+1}^{p}-r_{1} C_{n+1}^{p} E_{1, n+1}^{p}-r_{2} C_{n+1}^{p} E_{2, n+1}^{p}\right)+ \\
& \frac{h^{\alpha}}{\Gamma(\alpha+2)} \sum_{j=0}^{n} a_{j, n+1}\left(m C_{j}-r_{1} C_{j} E_{1, j}-r_{2} C_{j} E_{2, j}\right)
\end{aligned}
$$

The predictor $\mathrm{E}_{1, \mathrm{n}+1}^{\mathrm{p}}, \mathrm{E}_{2, \mathrm{n}+1}^{\mathrm{p}}, \mathrm{C}_{1, \mathrm{n}+1}^{\mathrm{p}}$ for Eq. 19 are based on:

$$
\begin{aligned}
& E_{1, n+1}^{p}=E_{1,0}+\frac{1}{\Gamma(\alpha)} \sum_{j=0}^{n} b_{j, n+1}\left(-d_{1} E_{1, j}+\frac{\left(C_{j}\right)^{2} E_{1, j}}{\left(C_{j}\right)^{2}+k_{1}}\right) \\
& E_{2, n+1}^{p}=E_{2,0}+\frac{1}{\Gamma(\alpha)} \sum_{j=0}^{n} b_{j, n+1}\left(-d_{2} E_{2, j}+\frac{\left(C_{j}\right)^{2} E_{2, j}}{\left(C_{j}\right)^{2}+k_{2}}\right) \\
& C_{n+1}^{p}=C_{0}+\frac{1}{\Gamma(\alpha)} \sum_{j=0}^{n} b_{j, n+1}\left(m C_{j}-r_{1} C_{j} E_{1, j}-r_{2} C_{j} E_{2, j}\right)
\end{aligned}
$$

The $a_{j}$ and $b_{j}$ in Eq. 19 and 20 can be calculated used the following formula:

$$
\begin{gathered}
a_{j, n+1}=\left\{\begin{array}{cc}
n^{\alpha+1}-(n-\alpha)(n+1)^{\alpha}, & j=0 \\
(n-j+2)^{\alpha+1}+(n-j)^{\alpha+1}-2(n-j+1)^{\alpha+1}, & 1 \leq j \leq n \\
1, & j=n+1
\end{array}\right. \\
b_{j, n+1}=\frac{h^{\alpha}}{\alpha}(n-j+1)^{\alpha}-(n-j)^{\alpha} 0 \leq j \leq n
\end{gathered}
$$

This computational scheme is very useful for numerical integration of fractional differential equations. Equation 19 and 20 are refereeing in the scheme discussed in Eq. 17 and 18.

\section{RESULTS AND DISCUSSION}

In this study, we discuss the numerical simulation of the model. First, we vary the values of $\alpha$, the fractional order from $1-0.25$. When $\alpha=1$ the model will become standard model. In Fig. 1 as $\alpha=1$, the results is similar to ordinary derivative model. There are periods when $\mathrm{C}$ and $\mathrm{E}_{1}$ grow together and there are periods when they interfere with each other. There are also periods when $\mathrm{C}$ and $\mathrm{E}_{1}$ die together. Futhermore, $\mathrm{E}_{2}$ is destroyed and has no effect on the growth of $\mathrm{C}$ and $\mathrm{E}_{2}$. The maximum value for tumor is $4 \mathrm{~mm}^{3}$. The numerical results used 100 days in their simulation in according to Rihan et al. (2016) (Table 2).

Figure 2 illustrates that as the value of reduces $\alpha$ from 1-0.75, the maximum possible value of tumor cells decreases. The maximum value now is $1.6 \mathrm{~mm}^{2}$. Also, the tumor cell volume damps with the passage of time. As $\alpha=0.5$, Fig. 3 shows that the tumor reaches to a constant value after 15 days but in Fig. 1 when the model is standard with $\alpha=0.5$ it was increasing with time (Fig. 1).

Figure 4 when $\alpha=0.25$ the result is similar to our results in the case of $\alpha=0.5$. In this case, we can see that all $\mathrm{E}_{1}, \mathrm{C}, \mathrm{E}_{2}$ converge to constant value. The maximum value for tumor is $0.4 \mathrm{~mm}^{2}$. Another important observation that the predictor-corrector method and Implicit Euler's method give the same results in all cases.

We presented a fractional-order model for tumor immune interactions. In this model, we provide a Holling type-III function and cross reactivity in fractional-order model for tumor-immune system interactions (Ahmed et al., 2012). Two immune effectors 

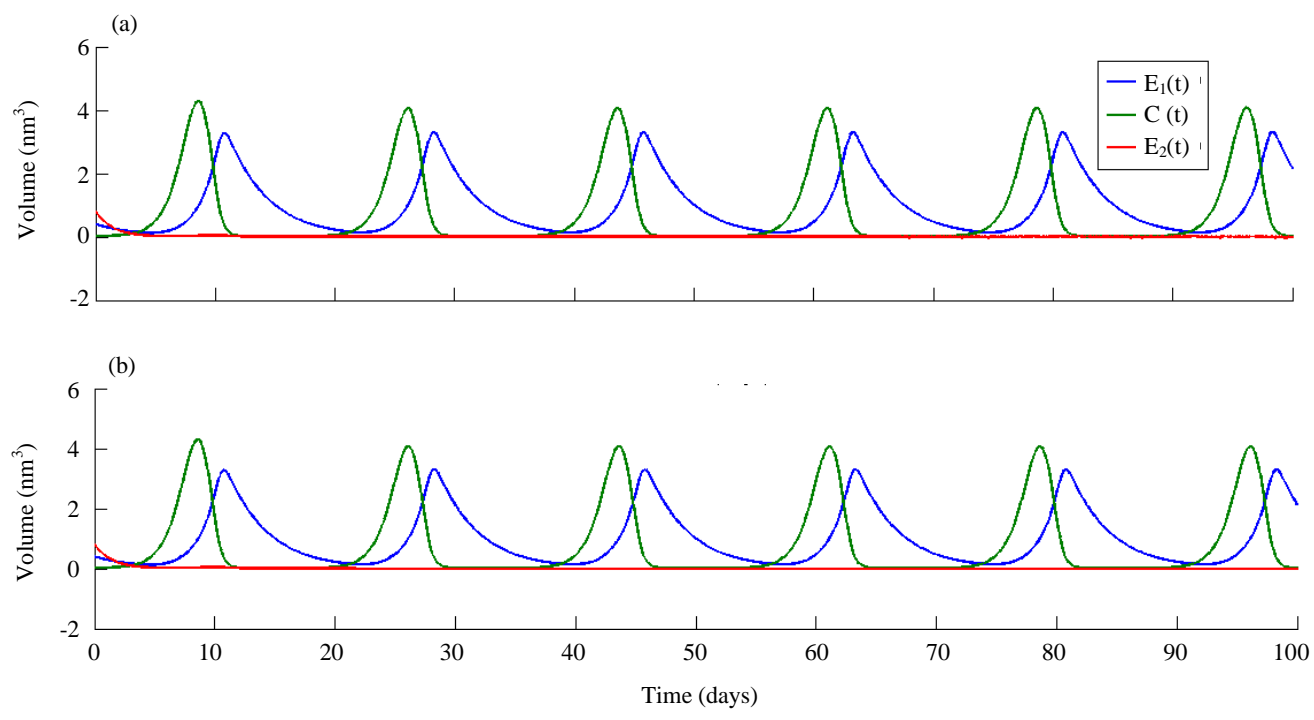

Fig. 1: Numerical results for Eq. 19 and 20 when $\alpha=1, d_{1}=0.3,0.7, d_{2}=0.7, k_{1}=0.3, k_{2}=0.7$ : a) Implicit Euler method and b) Predictor-corrector method)

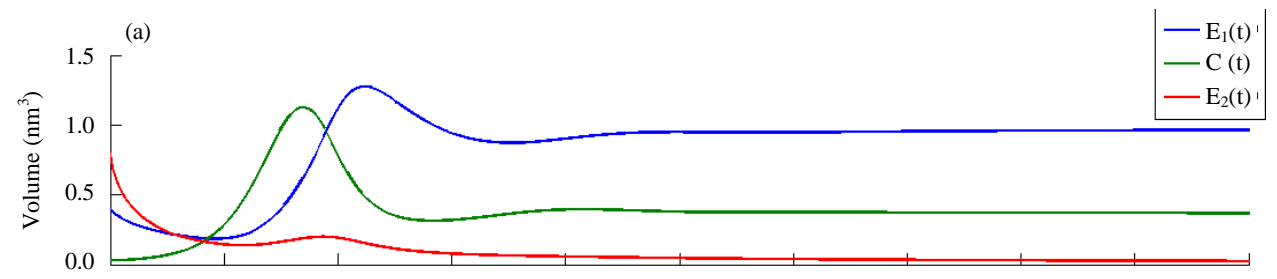

(b)

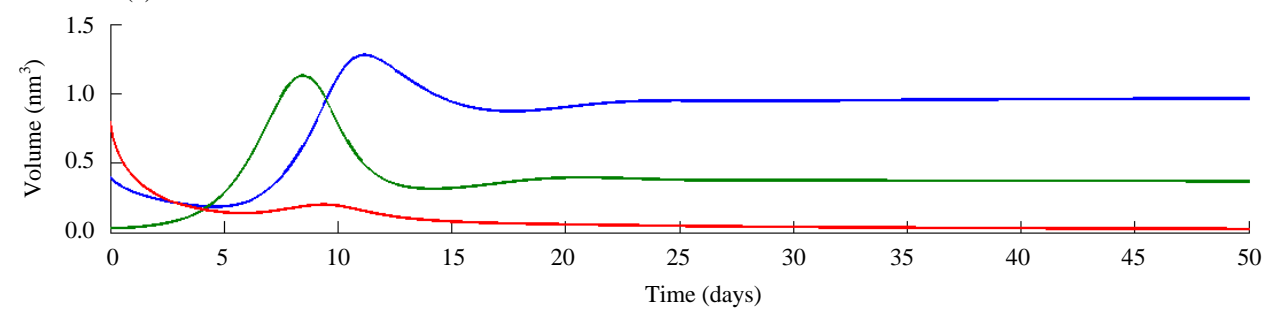

Fig. 2: Numerical results for Eq. 19 and 20 when $\left.\alpha=0.75, d_{1}=0.3,0.7, d_{2}=0.7, k_{1}=0.3, k_{2}=0.7: a\right)$ Implicit Euler method and b) Predictor-corrector method

Table 2: Numerical value for effector $E_{1}$, effector $E_{2}$ and tumor $C$ when $\alpha=1$

\begin{tabular}{llll}
\hline $\mathrm{n}$ & $\mathrm{E}_{\mathrm{l}}$ & $\mathrm{C}$ & $\mathrm{E}_{2}$ \\
\hline \multicolumn{2}{l}{ Implicit Euler's method } & & \\
1 & 0.4000 & 0.3000 & 0.8000 \\
100 & 0.1286 & 0.4795 & 0.0297 \\
200 & 2.4196 & 1.7878 & 0.0500 \\
300 & 1.0091 & 0.0049 & 0.0023 \\
400 & 0.22670 & 0.05396 & $7.15 \mathrm{e}-05$ \\
500 & 0.46187 & 2.86581 & $1.2 \mathrm{e}-05$ \\
Predictor-corrector method & & \\
1 & 0.4000 & 0.0300 & 0.8000 \\
100 & 0.1286 & 0.4794 & 0.0297 \\
200 & 2.4174 & 1.7970 & 0.0496 \\
300 & 1.0104 & 0.0050 & 0.0023 \\
400 & 0.227022 & 0.053934 & $7.06 \mathrm{e}-05$ \\
500 & 0.46152 & 2.86631 & $-3.06 \mathrm{e}-07$ \\
\hline
\end{tabular}

have been considered because of the property of multifunctions and multi-pathways of the immune system. In Fig. 1 as $\alpha=1$ the model becomes classical order where the tumor increase with time. However, reduction of $\alpha$ make a lot of changes to growth for tumor cells. We can see a robust feature of the fractional order modelling that the tumor growth decays (become a straight line). The maximum value for tumor is $4 \mathrm{~mm}^{2}$ (Fig. 1) and in the maximum value is $0.4 \mathrm{~mm}^{2}$ (Fig. 4). It is shown that a fractional model based on predictorcorrector scheme shows progression behavior result in accordance to the literature result by Rihan et al. (2016). 
(a)
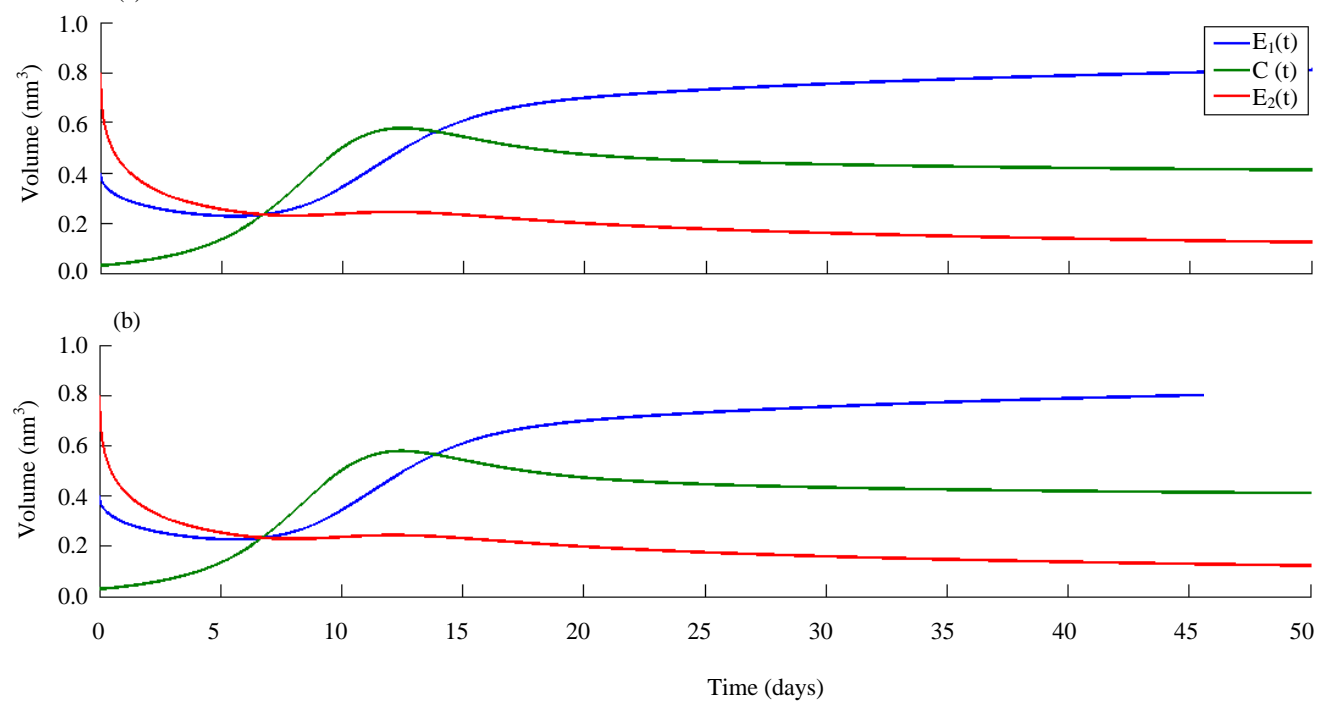

Fig. 3: Numerical results for Eq. 19 and 20 when $\alpha=0.5, d_{1}=0.3,0.7, d_{2}=0.7, k_{1}=0.3, k_{2}=0.7$ : a) Implicit Euler method and b) Predictor-corrector method

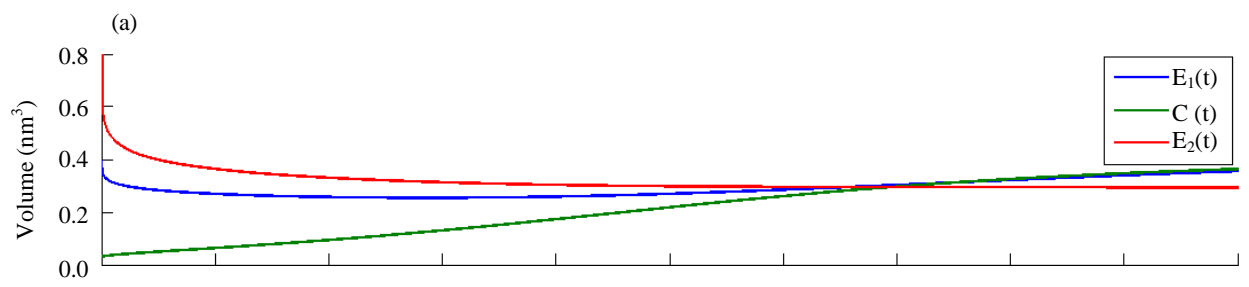

(b)

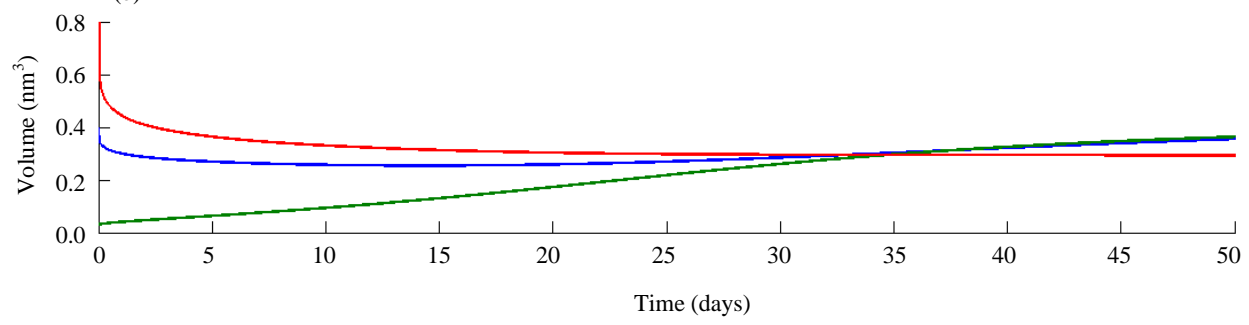

Fig. 4: Numerical results for Eq. 19 and 20 when $\alpha=0.25, d_{1}=0.3,0.7, d_{2}=0.7, k_{1}=0.3, k_{2}=0.7$ : a) Implicit Euler method and b) Predictor-corrector method

\section{CONCLUSION}

The numerical results from fractional-order derivatives system provide an excellent instrument for the description of hereditary properties related to inter and intra cells. However, in this research, we limit the description on hereditary properties towards cells behavior as our focus is more on adapting numerical scheme to the fractional tumor immune system. Numerical solution is carried out to confirm the analysis done by Rihan et al. (2016) by applying predictor-corrector method. The comparison results show that the predictor-corrector method simulation are in agreement with implicit Euler's method.

\section{REFERENCES}

Abdeljawad, T., 2011. On Riemann and Caputo fractional differences. Comput. Math. Appl., 62: 1602-1611.

Ahmed, E., A. Hashish and F.A. Rihan, 2012. On fractional order cancer model. J. Fractional Calculus Appl. Anal., 3: 1-6.

Ahmed, E.S.M. and H.A. El-Saka, 2010. On modeling two immune effectors two strain antigen interaction. Nonlinear Biomed. Phys., 4: 1-6.

Almeida, R., 2017. A Caputo fractional derivative of a function with respect to another function. Commun. Nonlinear Sci. Numer. Simul., 44: 460-481. 
Altrock, P.M., L.L. Liu and F. Michor, 2015. The mathematics of cancer: Integrating quantitative models. Nature Rev. Cancer, 15: 730-745.

Anonymous, 2018. Cancer. World Health Organization, Geneva, Switzerland. http://www.who.int/en/newsroom/fact-sheets/detail/cancer

Arshad, S., D. Baleanu, J. Huang, Y. Tang and M.M. Al Qurashi, 2016. Dynamical analysis of fractional order model of immunogenic tumors. Adv. Mech. Eng., 8: 113.

Bellomo, N. and L. Preziosi, 2000. Modelling and mathematical problems related to tumor evolution and its interaction with the immune system. Math. Comput. Model., 32: 413-452.

Bellomo, N., N.K. Li and P.K. Maini, 2008. On the foundations of cancer modelling: Selected topics, speculations and perspectives. Math. Mod. Methods Appl. Sci., 18: 593-646.

Caponetto, R., G. Dongola, L. Fortuna and I. Petras, 2010. Fractional Order Systems: Modeling and Control Applications. World Scientific, Singapore, ISBN13:978-981-4304-19-1, Pages: 177.

Diethelm, K., 2010. The Analysis of Fractional Differential Equations: An Application-Oriented Exposition using Differential Operators of Caputo Type. Springer, Berlin, Germany, ISBN:978-3-64214573-5, Pages: 253.
Diethelm, K., N.J. Ford and A.D. Freed, 2002. A predictorcorrector approach for the numerical solution of fractional differential equations. Nonlinear Dyn., 29: $3-22$.

Ferdi, Y., 2012. Some applications of fractional order calculus to design digital filters for biomedical signal processing. J. Mech. Med. Biol., 12: 1240008-11240008-13.

Iori, M., G. Nespi and G. Spiga, 1999. Analysis of a kinetic cellular model for tumor-immune system interaction. Math. Comput. Modell., 29: 117-129.

Itik, M. and S.P. Banks, 2010. Chaos in a threedimensional cancer model. Intl. J. Bifurcation Chaos, 20: 71-79.

Majid, Z.A. and M.B. Suleiman, 2006. 1-point implicit code of adams moulton type to solve first order ordinary differential equations. Chiang Mai J. Sci., 33: 153-159.

Mophou, G. and J.M. Fotsing, 2014. Optimal control of a fractional diffusion equation with delay. J. Adv. Math., 6: 1017-1031.

Rihan, F.A., 2013. Numerical modeling of fractional-order biological systems. Abstract Appl. Anal., 2013: 1-11.

Rihan, F.A., A. Hashish, F. Al-Maskari, M.S. Hussein and E. Ahmed, 2016. Dynamics of tumor-immune system with fractional-order. J. Tumor Res., 2: 1-6.

Sohail, A., S. Arshad, S. Javed and K. Maqbool, 2015. Numerical analysis of fractional-order Tumor model. Intl. J. Biomath., 8: 1550069-1-1550069-13. 